\title{
Training-induced plasticity in the visual cortex of adult rats following visual discrimination learning
}

\author{
Audrey M. Hager ${ }^{1}$ and Hans C. Dringenberg ${ }^{1,2,3,4}$ \\ ${ }^{1}$ Department of Psychology, Queen's University, Kingston, Ontario K7L 3N6, Canada; ${ }^{2}$ Centre for Neuroscience Studies, Queen's \\ University, Kingston, Ontario K7L 3N6, Canada; ${ }^{3}$ Department of Pharmacology and Toxicology, Queen's University, Kingston, \\ Ontario K7L 3N6, Canada
}

\begin{abstract}
Changes in synaptic efficacy, including long-term potentiation (LTP) and long-term depression (LTD), provide mechanisms for experience-induced plasticity and play a key role in learning processes. Some types of learning (e.g., motor learning, fear conditioning) result in LTP and/or LTD-like changes at synapses. Here, rats learned to discriminate two visual stimuli, P+ and $\mathrm{P}-$, indicating the presence and absence, respectively, of a hidden escape platform in a Y-shaped water maze. Following task acquisition, trained rats showed larger amplitude of visually evoked potentials (VEPs) in V1 to both stimuli encountered during training relative to novel stimuli. Training also resulted in a facilitation of LTP induced by theta-burst stimulation (TBS) of thalamic afferents to V1 with no effect on depression induced by low-frequency stimulation (LFS). Visual VEP enhancement and increased LTP both required that visual stimuli carried some significance to the animal, as both effects were absent in control rats exposed to the same visual stimuli in the absence of pairing with platform location. Together, these experiments show that visual experience can result in a stimulus-selective response enhancement and an increase in the synaptic modification range of V1 synapses, providing a novel example of metaplasticity in circuits of the adult cortex.
\end{abstract}

There is now considerable evidence suggesting that long-term potentiation (LTP) can serve as a mechanism mediating experience/training-dependent plasticity (Kirkwood et al. 1996; Rogan et al. 1997; Rioult-Pedotti et al. 2000; Whitlock et al. 2006). For example, training rats on a skilled forelimb reaching task resulted in an increase in strength of synapses in the primary motor cortex in vitro, which reduced the amount of LTP that could subsequently be induced using electrical high-frequency stimulation (Rioult-Pedotti et al. 2000). At the same time, training allowed greater levels of synaptic depression (long-term depression, LTD) induced by low-frequency stimulation (LFS), consistent with an up-regulation of intrinsic synaptic strength following learning. Similar findings have recently been obtained in vivo, showing an LTP-like increase in synaptic strength in the CA1 field of the hippocampus of rats after inhibitory avoidance learning (Whitlock et al. 2006). Again, this effect resulted in an occlusion of subsequent LTP induction, suggesting that similar plasticity mechanisms are engaged during learning and electrically induced LTP phenomena (Whitlock et al. 2006).

These and similar studies (e.g., Rogan et al. 1997) clearly imply up-regulation of synaptic efficacy as a potential mechanism mediating learning and memory-related functions in forebrain networks. However, recent evidence indicates that LTD-like processes may also occur following specific learning experiences. Massey et al. (2008) showed that the induction of LTD in the perirhinal cortex in vitro is occluded by previous, visual experience (viewing the same visual images repeatedly over $4 \mathrm{~d}$ ), while exposure to changing (novel) images had no effect. Consequently, LTP and LTD likely provide dual, complimentary mechanisms to adjust synaptic strength following various types of behavioral experiences.

\footnotetext{
${ }^{4}$ Corresponding author.
}

E-mail dringenb@queensu.ca; fax 613-533-2499.

Article is online at http://www.learnmem.org/cgi/doi/10.1101//m.1787110.
The notion that behavioral training can induce LTP-like changes at cortical and hippocampal synapses has been extended by showing that, in rodents, repeated exposure to visual stimuli (black and white gratings, checkerboards, light flashes) resulted in a potentiation of visually evoked field potentials (VEPs) recorded in the primary visual cortex (V1) under in vivo conditions (Clapp et al. 2006; Frenkel et al. 2006; Kuo and Dringenberg 2009); a similar phenomenon of sensory-induced LTP also occurs in humans (Teyler et al. 2005; Ross et al. 2008). The fact that potentiation of VEPs occurs for previously presented, but not novel, visual stimuli (Frenkel et al. 2006; Ross et al. 2008) suggests that sensory-induced LTP meets the criterion of input specificity, one of the hallmarks of classic LTP. Interestingly, in these studies, synaptic potentiation was induced in the absence of cognitive, attentional, or motivational demands on the subject; that is, mere, passive exposure was sufficient for these effects to occur. This finding is surprising, given that adult sensory cortices express resistance to synaptic modifications elicited by passive sensory exposure (e.g., De Villers-Sidani et al. 2007), which can only be overcome by introducing attentional and motivational demands (and the release of related neuromodulators) through behavioral learning procedures (Kilgard and Merzenich 1998; Bao et al. 2001; Weinberger 2004). At present, this discrepancy and the functional, behavioral significance of synaptic enhancement following repeated, passive exposure to visual stimuli remain to be determined.

With the present set of experiments, we investigated whether repeated exposure to visual stimuli in the context of a simple visual discrimination task results in changes in VEPs recorded in $\mathrm{V} 1$ of trained and untrained rats. The task required rats to discriminate two visual cues, one of which consistently indicated the location of a hidden escape platform in a modified, Y-shaped water maze apparatus (Fig. 1; also see Epp et al. 2008). Control animals were exposed to the same visual stimuli in the absence of explicit pairing of these stimuli with the platform location. This learning task provides a naturalistic paradigm to assess the 


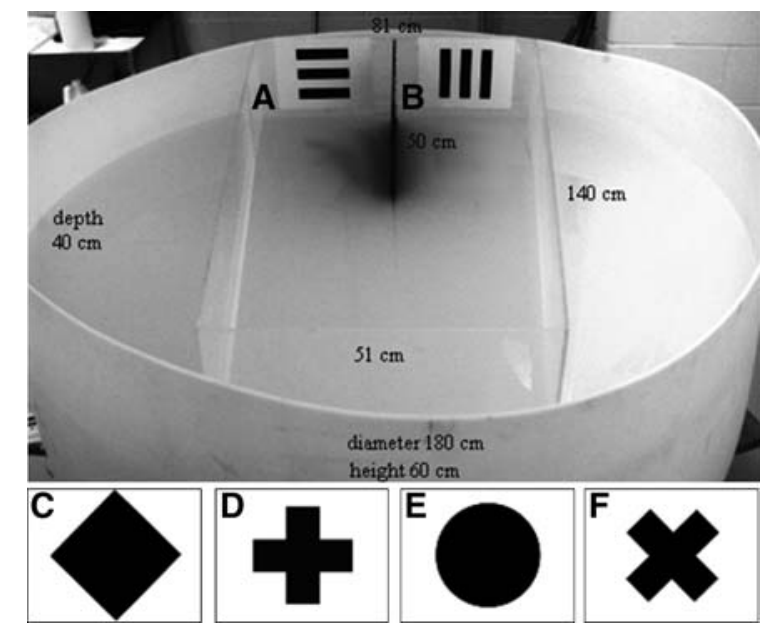

Figure 1. The Y-maze apparatus (including dimensions) and all visual cues used for visual discrimination training. $(A-C)$ Stimuli used for trained and control animals trained on one discrimination. For each animal, one of the stimuli served as P+ (indicating platform location), $\mathrm{P}$ - (no platform), and novel (all stimuli were counterbalanced among animals). $(D-F)$ Additional stimuli used for rats that received a second set of visual cues for extended visual discrimination training.

effect of visual experience on synaptic connectivity and plasticity in multiple brain areas, including V1. Following completion of the behavioral training, VEPs were recorded from the surface of V1 in response to familiar (encountered during the task) and novel visual stimuli. Further, the effect of visual experience on the induction of LTP and LTD (in separate animals) in V1 was assessed by theta-burst stimulation (TBS) and LFS, respectively, of the lateral geniculate nucleus (LGN). Shifts in LTP and/or LTD magnitude are a common measure to confirm changes in synaptic efficacy and modification range that may occur as a consequence of behavioral training (see Rioult-Pedotti et al. 2000, 2007; Whitlock et al. 2006).

\section{Results}

\section{Visual discrimination acquisition}

Rats that received training $(n=24)$ in the visual discrimination task required an average of $9 \mathrm{~d}$ (range 6-13 d) to reach the performance criterion of at least $80 \%$ correct trials per day for three consecutive days (Fig. 2). Two animals failed to successfully complete the task (did not reach criterion after $15 \mathrm{~d}$ of training) and were excluded from the study. As expected, control animals $(n=24)$ that underwent water maze training without predictive pairing of a visual cue with platform location failed to learn the task (Fig. 2); these animals entered the goal arm containing the escape platform on $\sim 44 \%$ of the trials. The lack of performance improvement in control rats indicates that distal, contextual cues in the test room were insufficient to locate the escape platform, and that the presence of local, visual cues (which were consistently associated with the changing platform location), was required for successful task acquisition.

\section{Effects of visual training on VEPs in task-naïve, control, and trained animals}

On the day following completion of the behavioral training, electrophysiological procedures were performed on trained and control animals. A further group of task-naïve animals also underwent all electrophysiological procedures (see below). For each rat, VEPs, recorded at the surface of V1, were elicited by the two familiar $(\mathrm{P}+, \mathrm{P}-)$ and one novel visual stimulus (100 presentations each; note that all three visual stimuli were novel for task-naïve animals). To examine possible changes in VEPs with repeated exposure to visual stimuli (see Frenkel et al. 2006), we computed averages of the first and last 100 VEPs in a recording session. These analyses did not reveal consistent changes in VEP amplitude as a result of repeatedly viewing the visual stimuli.

Typical VEPs consisted of a biphasic response, with an initial positive-going potential, followed by a negative-going deflection (Fig. 3A, inset). Latencies to the peaks of these two VEP components were $\sim 250 \pm 50 \mathrm{msec}$ and $700 \pm 50 \mathrm{msec}$, respectively. No electrical potentials were observed when a barrier was placed between the computer monitor and open eye of the rat, indicating that electrical or magnetic interference did not result in detectable voltage changes in V1 under these experimental conditions.

Initially, visual stimuli (Fig. 1A-C) were presented to tasknaïve animals $(n=21)$. For these rats, there were no significant amplitude differences in VEPs elicited by these stimuli (repeated-measures ANOVA; $F_{(2,40)}=0.11 ; P>0.05$ ), indicating that the different elements and design of visual stimuli themselves had no major influence on VEP responses (Fig. 3A).

Next, VEPs (elicited by two familiar and one novel visual stimulus) were recorded in control animals $(n=24)$ that had undergone swimming and exposure to visual stimuli, but without pairing of visual cues and platform location. As was the case in task-naïve animals, control rats showed similar VEP amplitudes for all visual stimuli (Fig. 3B), irrespective of whether or not they were seen during the swim task (repeated-measures ANOVA; $\left.F_{(2,46)}=0.68 ; P>0.05\right)$. Consequently, mere passive exposure to visual stimuli during training was not sufficient to influence cortical VEPs relative to completely novel exemplars.

Finally, VEP procedures were carried out in rats that had completed the visual discrimination learning $(n=24)$. In these animals, comparisons of VEPs elicited by P+ (indicating the maze arm containing the platform), $\mathrm{P}-$ (indicating the maze arm without platform), and a novel stimulus revealed a significant effect of visual stimulus on VEP amplitude (Fig. 3C; repeated-measures ANOVA; $\left.F_{(2,46)}=3.25 ; \quad P<0.05\right)$. Pairwise comparisons of VEPs triggered by $\mathrm{P}+, \mathrm{P}-$, or a novel stimulus using LSD (least significant differences) showed greater amplitude of $\mathrm{P}+\mathrm{VEPs}$ relative to novel VEPs (Fig. 3A; $P=0.031$ ), while the difference between $\mathrm{P}-$ VEPs and novel VEPs approached

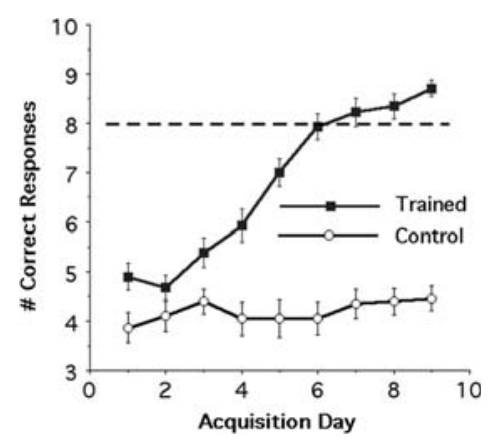

Figure 2. Average ( \pm S.E.M.) number of correct $Y$-maze arm entries during visual discrimination training. Data show correct responses for both trained $(n=24$; receiving a consistent association between visual cues and platform location) and control animals $(n=24$; receiving a random association between visual cues and platform location). (Dashed line) Criterion set for successful completion of the task. For both groups, correct trials were scored when a rat entered the correct goal arm and mounted the platform; errors were scored when a rat entered the incorrect goal arm (i.e., not containing the platform) with at least half of its body. 




Figure 3. Amplitude ( \pm S.E.M.) of visual evoked potentials (VEPs) in task-naïve rats $(A)$, swim controls $(B)$, and rats receiving visual discrimination training $(C, D)$. $(A)$ There were no significant differences in VEP amplitude among the three visual stimuli in task-naïve rats $(n=21$; note that all cues are novel to these animals). (Inset) A typical VEP recorded at the cortical surface of $\mathrm{V} 1$ in a urethane-anesthetized rat (VEP shown is an average of 100 individual sweeps; calibration bars are $0.2 \mathrm{mV}$ and $100 \mathrm{msec}) .(B)$ Control rats $(n=24)$ that underwent swimming without visual stimuli predicting the platform location also showed no significant difference among VEPs elicited by stimuli present in the maze, and a novel stimulus not encountered during training. (Left, Right) Stationary position of the stimuli in the $\mathrm{Y}$ maze during swim training. (C) Animals that underwent visual discrimination training $(n=$ 24) and learned to reliably distinguish between $P+$ (indicating platform presence) and $\mathrm{P}$ - (absence of platform) showed greater VEP amplitude to stimuli encountered during training compared with novel stimuli, an effect that was significant for $\mathrm{P}+(*, P<0.05)$ and approached significance for $\mathrm{P}-$. (Overlays) VEP amplitudes elicited by the three visual stimuli presented to each individual animal. Note that S.E.M. error bars represent variance between animals, but the statistical model consists of repeated-measures, within-subject analysis of variance (ANOVA) comparing VEPs elicited by the three visual stimuli presented to the same animal. $(D)$ The same data as in panel $C$ are plotted, but collapsing across the two familiar visual stimuli encountered during training $(P+$ and $P-)$. VEPs elicited by the familiar stimuli were significantly $(*, P<0.05)$ larger than those elicited by a novel stimulus (see note in panel $C$ for comments regarding the appropriate interpretation of S.E.M. error bars).

significance $(P=0.079)$; VEPs elicited by $\mathrm{P}+$ and $\mathrm{P}-$ were not different (Fig. $3 C ; P=0.689)$. When familiar stimuli $(\mathrm{P}+$ and $\mathrm{P}-$ ) were pooled and compared with novel stimuli, there was a significant difference, with larger-amplitude VEPs seen for familiar stimuli (Fig. 3D; repeated-measures $t$-test; $t_{(23)}=2.26 ; P=0.034$ ). Consequently, it appears that visual discrimination learning results in a selective enhancement of V1 responses elicited by behaviorally significant cues encountered during training, while mere exposure to visual stimuli is insufficient to enhance synaptic responses under the present, experimental conditions.

\section{Effects of visual training on the modification range of $\mathrm{Vl}$ synapses}

Following the completion of VEP recordings, the LGN stimulation electrode was inserted to evoke field postsynaptic potentials
(fPSPs) in V1 (see Hager and Dringenberg [2010] for a figure depicting a typical LGN electrode location). Consistent with previous observations (Heynen and Bear 2001; Dringenberg et al. 2007), single-pulse stimulation of the LGN elicited negative-going fPSPs recorded at the surface of the ipsilateral V1 (Fig. 4, insets). These potentials largely reflect current sinks originating in layers II/III of the rat V1 (Heynen and Bear 2001).

To determine whether visual discrimination training alters levels of synaptic depression or potentiation in V1, separate groups of animals received either TBS or LFS of the LGN. In task-naïve rats $(n=7)$, two episodes of TBS (60 min apart) elicited reliable, synaptic potentiation, with fPSP amplitude at $140 \%$ of baseline during the last $30 \mathrm{~min}$ of the experiment (Fig. 4A). A similar magnitude of LTP was observed in control rats $(n=10)$, which showed an increase to $147 \%$ of baseline (Fig. 4A). However, trained rats $(n=7)$ showed significantly greater synaptic enhancement, with levels of LTP at $171 \%$ of baseline during the last $30 \mathrm{~min}$

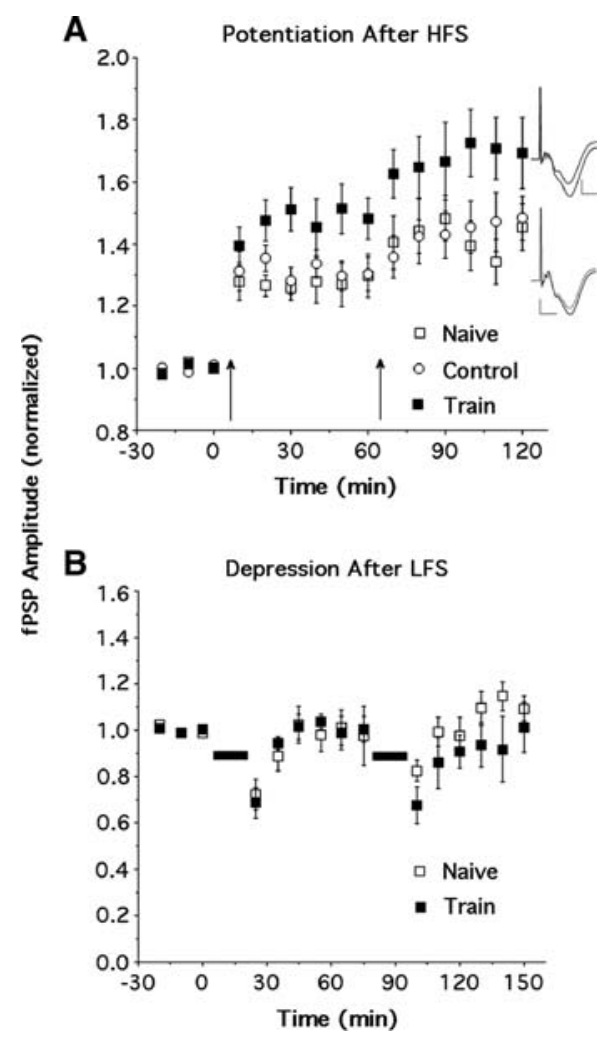

Figure 4. $(A)$ Amplitude $( \pm$ S.E.M.) of field postsynaptic potentials (fPSPs) before and after theta-burst stimulation (TBS, at arrows; $A$ ) or lowfrequency stimulation (LFS, at bars; $B$ ) of the lateral geniculate nucleus (LGN). (A) Thalamic TBS (five pulses/burst at $100 \mathrm{~Hz}$, repeated at $5 \mathrm{~Hz}$ ) induced LTP in all groups: task-naïve $(n=7)$, swim control $(n=10)$, and animals that underwent visual discrimination training $(n=7)$. However, the level of potentiation was greater in trained animals compared with the other two groups $(P<0.05)$. Normalized amplitude during the final $30 \mathrm{~min}$ of the experiment for each group was: task-naive $=1.40$, control $=1.47$, and trained $=1.71$ of baseline. $(B)$ Amplitude of fPSPS ( \pm S.E.M.) showed a small, transient depression immediately after LFS (900 bursts repeated at $1 \mathrm{~Hz}$, three pulses/burst at $20 \mathrm{~Hz}$, black bars), which recovered to baseline levels within $20 \mathrm{~min}$, an effect that was similar in task-naïve $(n=5)$ and trained $(n=6)$ animals. (Insets) Typical fPSPs in V1 in response to single-pulse stimulation of the LGN before (gray) and after (black) TBS in trained (top) and control (bottom) animals (each fPSP shown is an average of the first and last $30 \mathrm{~min}$ of recording, calibration is $0.25 \mathrm{mV}$ and $10 \mathrm{msec}$; note the greater potentiation in the trained animal). 
of recordings (Fig. 4A). A mixed, two-way ANOVA revealed main effects of time $\left(F_{(14,294)}=52.03 ; P<0.01\right)$ and group $\left(F_{(2,21)}=\right.$ $3.56 ; P<0.05)$, as well as an interaction of time $\times$ group $\left(F_{(28,294)}=1.80 ; P<0.01\right)$. Follow-up ANOVAs comparing trained with either control or task-naïve animals revealed significant time by group effects for both analyses (trained compared with controls: main effect of time $\left[F_{(14,210)}=40.39 ; P<0.01\right]$ and group $\left[F_{(1,15)}=4.89 ; P<0.05\right]$ as well as an interaction of time $\times$ group $\left[F_{(14,210)}=2.19 ; P<0.01\right]$; trained compared with naïve: main effects of time $\left[F_{(14,168)}=38.95 ; P<0.01\right]$ and group $\left[F_{(1,12)}=\right.$ 4.97; $P<0.05]$ interaction of time $\times$ group $\left[F_{(14,168)}=2.79 ; P<\right.$ 0.01]). Overall, these results indicate that greater levels of LTP emerged in trained animals over the course of the experiment (Fig. 4A).

In a separate group of naïve animals $(n=5)$, application of two episodes (60 min apart) of LFS to the LGN resulted in transient depression of fPSP amplitude, which typically recovered to baseline level within $20 \mathrm{~min}$ after the stimulation (Fig. 4B); results consistent with previous observations (Hager and Dringenberg 2010). Trained rats $(n=6)$ receiving LFS showed a similar pattern of transient fPSP depression that was not significantly different from that seen in naïve animals (Fig. 4B; mixed two-way ANOVA; main effects of time, $F_{(14,126)}=6.23 ; P<0.01$ and group, $F_{(1,9)}=0.59 ; P>0.05 ;$ interaction, $\left.F_{(14,126)}=1.09 ; \quad P>0.05\right)$. Together, these data indicate that visual discrimination training resulted in a change in the overall synaptic modification range by means of facilitation of LTP, without concurrent effects on synaptic depression of V1 synapses.

\section{Rats receiving two sets of visual discrimination training}

A final group of rats $(n=9)$ underwent extended behavioral training by first requiring them to discriminate one set of visual cues (same procedure as above), which was followed by further training using a second (new) cue set. Rats were divided into two subgroups to counterbalance the first (Fig. 1A-C) and second set of visual cues (Fig. 1D-F). The average number of days to reach the performance criterion $(80 \%$ correct for three consecutive days) for the first set of cues was $8.7 \mathrm{~d}$, similar to the results reported above (trained group). The average number of days to reach the performance criterion for the second set of cues was $5.1 \mathrm{~d}$, which was significantly shorter than the first training period (Fig. 5A; repeated-measures $t$-test, $t_{(8)}=2.35 ; P<0.05$ ). Even during the first two days of training on the second cue set, rats performed at $\sim 70 \%$ correct (Fig. $5 \mathrm{~A}$ ), reflecting very rapid, withinsession learning of the new visual cues (for the first day of training on the second cue set: group averages of $\sim 55 \%$ and $90 \%$ correct for the first and last [tenth] training trial, respectively).

To assess whether acquisition of the second cue set interfered with the retention of the first set, a probe test (10 trials) using the first set of cues was conducted $1 \mathrm{~d}$ following successful completion of the second training phase. For this test, eight of nine rats met or exceeded the performance criterion of $80 \%$ correct trials (average of 90\% correct; data not shown).

Finally, we examined whether extended training resulted in even greater facilitation of LTP elicited by TBS of the LGN. However, levels of potentiation induced by two TBS episodes (60 min apart) were equivalent to those seen in rats that had received training on only one set of visual cues (Fig. 5B; mixed two-way ANOVA main effect of time, $F_{(14,182)}=24.43 ; P<0.05$; group, $F_{(1,13)}=0.03 ; P>0.05$; interaction, $F_{(14,182)}=0.49 ; P>$ 0.05). Thus, the enhancement of LTP by visual discrimination training appears to be maximal after completion of the initial learning set.

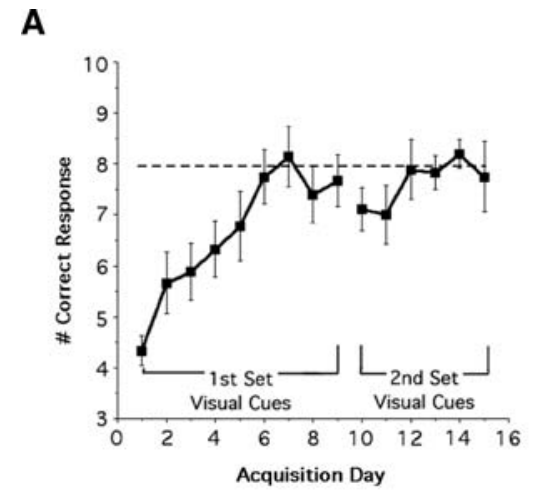

B

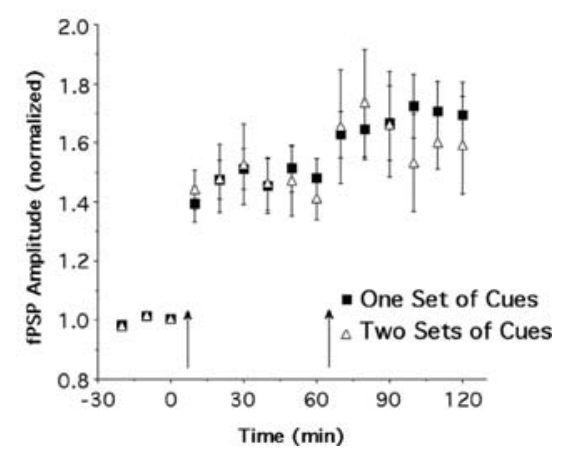

Figure 5. (A) Average ( \pm S.E.M.) number of correct arm entries in the Y-maze for animals $(n=9)$ that learned to discriminate two sets of visual cues. Acquisition of the second set of cues occurred significantly more quickly than initially learning of the first cue set $(P<0.05)$. (Dashed line) Criterion set for successful completion of the task. (B) Amplitude ( \pm S.E.M.) of field postsynaptic potentials (fPSPs) before and after theta-burst stimulation (TBS, at arrows) of the lateral geniculate nucleus (LGN) in rats trained on two sets of visual discrimination cues $(n=8)$ and animals trained on only one cue $(n=7$; same data as shown in Fig. 4A). Thalamic TBS induced similar levels of synaptic potentiation in both groups of animals.

\section{Discussion}

The present study demonstrates that visual discrimination learning results in stimulus selective facilitation of neuronal responses at early stages of visual processing (V1). In trained animals, familiar stimuli encountered during training elicited larger VEPs than novel stimuli. Control animals receiving (passive) exposure to the stimuli did not exhibit VEP enhancements to stimuli encountered during training relative to novel stimuli. Thus, it appears that the enhanced neuronal responsiveness seen here requires stimuli to carry some behavioral significance (e.g., indicating the platform location). Surprisingly, visual discrimination learning did not occlude the subsequent induction of LTP in V1; rather, it altered the plasticity properties of V1 neurons by facilitating LTP, with no apparent effect on LTD. Consequently, the synaptic modification range in V1 was increased following visual discrimination training.

The present experiments confirm that a significant degree of plasticity is present in the mature V1 of adult rodents that can be detected as a result of visual discrimination learning. Animals trained with a consistent platform-stimulus association exhibited enhanced amplitude VEPs elicited by familiar compared with novel visual stimuli. The VEPs analyzed here, recorded at or close to (i.e., layer I) the cortical surface, consisted of an initial, positive-going potential, similar to those seen in previous work 
(Heynen and Bear 2001). Using current source density analysis, Heynen and Bear (2001) showed that these potentials reflect a superficial current source, which is driven by an active current sink in layers II/III. Consequently, the change in VEP amplitude seen here could result from a greater number of layers II/III neurons activated when viewing familiar stimuli, or a stronger depolarization of the same set of neurons. Future work employing intracellular recordings of V1 neurons, or extracellular ensemble recordings of large groups of V1 cells, would be useful to further characterize the underlying, cellular mechanisms mediating this effect.

Similar to our observations, previous work has shown that repeated exposure to the same visual stimuli can result in an enhancement of VEPs in the V1 of rodents (Clapp et al. 2006; Frenkel et al. 2006; Kuo and Dringenberg 2009) and humans (Teyler et al. 2005; Ross et al. 2008). Surprisingly, the enhancement effects reported in these studies were seen with mere passive exposure to visual stimuli, in the absence of explicit behavioral training. For example, Frenkel et al. (2006) noted stimulusselective response enhancements in V1 of nonanesthetized, restrained mice following daily (3- to 4-d) exposure to sine-wave (bar) gratings or black-and-white checkerboards. In contrast, we noted that VEP enhancements occurred only in animals required to associate distinct visual cues with the presence and absence of the escape platform, while control animals that encountered nonpredictive (i.e., not associated with the platform) visual stimuli did not exhibit this effect. A likely reason for this apparent discrepancy lies in the fact that our control rats encountered visual stimuli as part of a larger, complex visual scene (the entire test room and apparatus), but visual cues within this scene carried no instructive significance for completing the behavioral task. As a consequence, control rats likely direct little or no attentional resources to the visual stimuli. As such, the pattern of results obtained here is consistent with previous studies demonstrating the requirements for attentional, motivational processes to elicit synaptic modifications in adult, fully mature sensory cortices (Kilgard and Merzenich 1998; Bao et al. 2001; Weinberger 2004).

Several recent investigations have demonstrated that learning experiences can limit or occlude the subsequent induction of LTP; that is, there is a trade-off between behaviorally induced synaptic potentiation and electrically induced LTP in neocortical and subcortical systems (motor cortex, hippocampus) (Whitlock et al. 2006; also see Rogan et al. 1997). Consequently, we were surprised to observe significantly greater levels of LTP in trained rats relative to control and task-naïve animals. This apparent discrepancy might, at least in part, be related to different methodological approaches. For example, occlusion of LTP in the motor cortex after motor learning was demonstrated at local, intracortical synapses of in vitro slices (Rioult-Pedotti et al. 2000), while we employed in vivo preparations and assessed synaptic strength in a polysynaptic pathway (see above). Whether training affected only a portion of this projection system is currently unknown. However, the clear facilitation of LTP as a result of visual learning indicates that synapses along this polysynaptic pathway between LGN and superficial V1 do not appear to be in a potentiated state. Thus, strengthening of these synapses might not serve as a mechanism for the long-term storage of specific visual stimuli encountered during training. Recently, using a discrimination task similar to that employed here, Epp et al. (2008) showed that hippocampal damage in rats results in a severe memory loss for previously learned visual stimuli. Importantly, this memory loss occurred even for long delays (60 d) between task acquisition and the lesioning procedure, suggesting that the hippocampus plays a critical role in the longterm storage of visual memories (Epp et al. 2008).
The facilitation of LTP noted here suggests that, rather than acting as a storage mechanism, the upper limits of potentiation of V1 synapses are raised, an effect that appears to occur without concurrent changes in the levels of synaptic depression. In other words, there is an increase in the synaptic modification range in V1 following visual discrimination training. To date, very few studies have examined changes in the synaptic modification range following behavioral experiences. As mentioned above, motor learning results in a LTP-like synaptic strengthening in the primary motor cortex of rats, which limits the amount of LTP that can subsequently be induced by electrical stimulation (Rioult-Pedotti et al. 2000). Interestingly, this enhancement in synaptic strength is maintained for several months after training is discontinued. However, the entire range of synaptic modifications that can be elicited in the same set of synapses shift upward. In other words, the ceiling of LTP is raised, allowing further potentiation of the previously strengthened synapses. This "renormalization" of synaptic strength in the center of the modification range could support further synaptic enhancements induced by novel learning experiences (Rioult-Pedotti et al. 2007). The results of Rioult-Pedotti et al. (2007) differ from those observed here in that we did not detect a shift, but rather an expansion of the modification range (unchanged LTD, enhanced LTP). This use-dependent enhancement of synaptic plasticity in V1 could play a role in, and facilitate processes related to, perceptual learning such as visual acuity (see Prusky et al. 2000b) occurring in V1, rather than the associational, cue-specific learning mediated by hippocampal circuits.

It is of interest to note that rats receiving extended visual discrimination training learned to discriminate a second set of visual cues much more readily than the initial set. Such an effect could be due to several mechanisms operating in parallel, including the acquisition of a general "schema," learning of general task requirements (i.e., procedural learning such as swimming toward the goal arms, using the specific visual cues in the maze as a guide), and perhaps perceptual learning, that is, an improvement of basic, perceptual functions (e.g., visual acuity, contrast sensitivity, binocular integration) carried out by V1 circuits.

Recent data have demonstrated that, in adult rodents, environmental enrichment results in an enhancement of white matter stimulation-induced LTP measured in layers II/III of V1 slices (Sale et al. 2007). This enrichment-induced enhancement of plasticity is similar to the greater LTP we noted following visual discrimination training, which clearly provides a form of visual enrichment to rodents, even though it is surprising that control animals did not exhibit a similar effect. The fact that environmental enrichment leads to the development of greater visual acuity (Prusky et al. 2000a) and promotes the recovery of degraded (by monocular deprivation in early life) visual acuity (Sale et al. 2007) suggests that the greater plasticity range in V1 might have a functional significance for the improvement of perceptual functions at the early stages of visual, cortical processing. It is tempting to speculate that such mechanisms might mediate, at least in part, the facilitation of performance noted here with extended visual training, a hypothesis that requires further tests for its critical evaluation.

At present, the cellular and neurochemical mechanisms mediating the stimulus-specific VEP enhancement, as well as the shift in LTP ceiling, remain to be determined. A considerable body of evidence suggests that acetylcholine (ACh) plays a critical role in the induction and facilitation of plasticity in primary sensory cortices (Rasmusson 2000; Gu 2003; Weinberger 2004). For example, in the primary auditory cortex, pairing the release of endogenous ACh with an acoustic stimulus of a specific frequency is sufficient to increase the area of A1 responsive to those tones (Kilgard and Merzenich 1998), and a similar, 
ACh-dependent effect occurs with behavioral auditory discrimination training (Weinberger 2003, 2004). These effects are reminiscent of the enhanced VEP response to stimuli that play an instructive role during visual discrimination learning, raising the possibility that ACh mediates the VEP enhancements observed here (also see Fournier et al. 2004; Kang and Vaucher 2009; Rodriguez et al. 2010). Similarly, the mechanisms underlying the greater levels of LTP in V1 induced by LGN stimulation are currently unknown, but could involve several parallel cellular and neurochemical systems, including the release of trophic factors (e.g., BDNF) during training, changes in the subunit composition of NMDA receptors (NR2A, NR2B), or reductions in inhibitory GABAergic activity, all of which have been shown to act as potent regulators of plasticity at V1 synapses (Hanover et al. 1999; Jiang et al. 2005; Karmarkar and Dan 2006; Yashiro and Philpot 2008). Clearly, further work is necessary to characterize the cellular and neurochemical systems involved in the various effects demonstrated in the present experiments.

In summary, the data presented here show that visual discrimination training results in a stimulus-selective facilitation of neuronal responses in V1. Passive (noninstructive) exposure to the same stimuli did not elicit a similar effect, indicating that this effect is selective for task-relevant, biologically significant sensory information. Further, and contrary to previous work (Rioult-Pedotti et al. 2000; Whitlock et al. 2006), we noted greater levels of LTP in V1 in vivo following visual discrimination learning, indicative of an expanded synaptic modification range as a result of the training experience. Animals required to learn multiple visual discriminations acquired the second discrimination set much more readily. Whether the enhanced plasticity range in $\mathrm{V} 1$ plays a role in this performance facilitation, and the underlying synaptic and neurochemical mechanisms mediating the effects reported here, remain to be determined. Thus, visual discrimination training can alter the ability of V1 neurons to undergo synaptic potentiation, providing a further, novel example of metaplasticity in circuits of the adult mammalian cortex.

\section{Materials and Methods}

\section{Subjects}

All experiments were conducted in accordance with published guidelines of the Canadian Council on Animal Care and approved by the Queen's University Animal Care Committee. The experiments were conducted on adult (250-600 g), male Long-Evans rats obtained from Charles River Laboratories, Inc. (St. Constant, Quebec, Canada). Animals were housed in a colony room (12/ $12 \mathrm{~h}$ reversed light cycle) with free access to food and water. All behavioral tests and electrophysiological procedures took place during the day, typically between the time of 9:00 and 17:00 $\mathrm{h}$.

\section{Y-maze visual discrimination apparatus}

Visual discrimination training was performed in a modified water maze, which included a Y-maze insert and distinct visual cues. The water maze (Fig. 1) was a circular pool (diameter $180 \mathrm{~cm}$, height $60 \mathrm{~cm}$ ) made of white, opaque Perspex. It was filled with water $\left(21^{\circ} \mathrm{C} \pm 1^{\circ} \mathrm{C}\right)$ to a depth of $40 \mathrm{~cm}$. The Y-maze insert (height $61 \mathrm{~cm}$, length $140 \mathrm{~cm}$, width $51 \mathrm{~cm}$, and $81 \mathrm{~cm}$ ), constructed of clear Plexiglas, was placed inside the water maze with the same orientation for all trials. A black divider (length $50 \mathrm{~cm}$, height $61 \mathrm{~cm})$ separated the two goal arms. The rectangular platform (width $12 \mathrm{~cm}$, height $38 \mathrm{~cm}$, length $36 \mathrm{~cm}$ ) of clear Plexiglas was positioned $10 \mathrm{~cm}$ from the end of one of the two goal arms, at $\sim 2 \mathrm{~cm}$ below the water surface. Nontoxic white tempera paint was added to the water to ensure that the platform was not visible. The fluorescent lighting in the room was muted to avoid glare on the pool surface and visual cues.

\section{Visual cues}

Computer-generated visual cues were printed on white sheets of paper $(28 \mathrm{~cm} \times 21.5 \mathrm{~cm})$. The following six visual cues were used (Fig. 1; labeled A-F from hereon): (A), three equally spaced black horizontal bars (length $15 \mathrm{~cm}$, width $3 \mathrm{~cm}$, spaced $3 \mathrm{~cm}$ apart, black area $135 \mathrm{~cm}^{2}$ ); (B), three equally spaced black vertical bars (same dimensions and spacing); (C), a black diamond $\left(15 \mathrm{~cm} \times 15 \mathrm{~cm}\right.$, area $\left.225 \mathrm{~cm}^{2}\right)$; (D), a black plus symbol (area $135 \mathrm{~cm}^{2}$ ); (E), a black circle (diameter $16.7 \mathrm{~cm}$, area $222 \mathrm{~cm}^{2}$ ); and (F), a black plus sign rotated by $45^{\circ}\left(\right.$ area $\left.135 \mathrm{~cm}^{2}\right)$. Cues were mounted at the ends of the goal arms $1 \mathrm{~cm}$ above the water surface.

\section{Visual discrimination training}

Visual discrimination training was divided into three phases. For all phases, animals were released into the maze facing the pool wall and required to swim toward the goal arms in order to reach the hidden escape platform.

\section{Phase 1}

The initial training phase ( $1 \mathrm{~d})$ served to familiarize and habituate rats to swimming in the pool and finding the hidden platform without the use of explicit visual cues in the maze. Rats were released into the pool and given a maximum of $200 \mathrm{sec}$ to explore the maze and find the hidden platform in one of the goal arms. If a rat failed to do so, it was manually guided to the platform by the experimenter. Animals remained on the platform for $15 \mathrm{sec}$ before commencement of the next trial. Trials were repeated (platform remained in the same arm) until a rat performed five consecutive correct responses, or a maximum of 10 trials, whichever came first (this constituted a "trial block"). Correct trials were scored when a rat entered the correct goal arm and mounted the platform; errors were scored when a rat entered the incorrect goal arm with at least half of its body.

Each trial block was followed by a 5 -min rest period, where rats were returned to a temporary holding cage with holes in the bottom to allow water to drain. Following the rest period, the next trial block was administered using the same procedures as above, with the exception that the platform was moved to the opposite goal arm. A total of four trial blocks were administered, each followed by a 5-min rest period. After completion of training, animals were placed under a heat lamp for a minimum of $15 \mathrm{~min}$ before being returned to their home cage.

\section{Phase 2}

The same training procedure as that outlined above was used for Phase 2 ( $1 \mathrm{~d})$, with the exception that two distinct visual cues were placed at the end of the goal arms, one indicating the presence of the platform $(\mathrm{P}+)$, while the other cue indicated its absence $(\mathrm{P}-)$. Again, platform location and visual cues remained unchanged during each trial block, but alternated between blocks, keeping the platform-cue association constant for all blocks. However, for a group of control animals, the platform location alternated between blocks, while the visual cues remained constant to ensure that they did not predict the platform location. The two cues employed during behavioral training where chosen from cues A-C listed above (see Fig. 1) while the third was used as a "novel exemplar" during subsequent VEP recordings (see below). The assignment of cues as $\mathrm{P}+, \mathrm{P}-$, and novel was counterbalanced across different animals.

\section{Phase 3}

The final training phase commenced on day 3 and continued until rats reliably discriminated the two visual cues. Procedures were the same as for Phase 2, with the following exceptions. For each day, rats received 10 training trials, each followed by a 30 -sec rest period in a holding cage. For each trial, platform location was pseudorandomly assigned to one goal arm, with the restriction that each arm contained the platform on five out of 
10 trials. Again, for one group of rats, the $\mathrm{P}+$ and $\mathrm{P}-$ cues consistently indicated the platform presence and absence, respectively. For the control group, platform location changed pseudorandomly from trial to trial, but visual cues remained stationary, thus lacking a consistent association with the platform. For cuetrained rats, daily training continued until a rat reached a criterion of at least $80 \%$ correct (i.e., eight of 10 daily trials) over three consecutive days. For control animals, daily swimming was carried out for nine consecutive days, the average time required by cue-trained rats to reach the performance criterion outlined above. Throughout training, the experimenter manually recorded platform location, as well as correct and incorrect responses for all animals. Again, for both visually trained and control rats, correct trials were scored when a rat entered the correct goal arm and mounted the platform; errors were scored when a rat entered the incorrect goal arm (i.e., not containing the platform) with at least half of its body.

\section{Multiple visual cue discrimination}

An additional group of animals received visual discrimination training during Phases $1-3$. However, after successful completion of Phase 3, training continued using two novel visual cues, acting as $\mathrm{P} 2+$ and $\mathrm{P} 2-$ (two of cues D-F). The same procedure as in Phase 3 was carried out, until the performance criterion was reached. On the final test day, a probe trial was conducted with the original set of visual cues $(\mathrm{P}+$ and $\mathrm{P}-)$ in order to assess whether the extended training period and/or subsequent learning influenced the retention of the original set of visual cues.

\section{Surgical preparation}

Electrophysiological procedures were performed on the day following completion of training, with rats under deep urethane anesthesia $(1.5 \mathrm{~g} / \mathrm{kg}$, intraperitoneal [i.p.], administered in three $0.5 \mathrm{~g} / \mathrm{kg}$ doses every $20 \mathrm{~min}$ ). Typically, urethane supplements were in the range of an additional $0.5 \mathrm{~g} / \mathrm{kg}$ and were administered prior to the onset of data collection. This dosing regiment is sufficient to ensure that spontaneous high-frequency, low-amplitude activation in the electrocorticogram does not occur during the course of the experiment (Kuo and Dringenberg 2008). Additionally, the local anesthetic Marcaine $(5 \mathrm{mg} / \mathrm{ml}$, subcutaneous [s.c.], three to four injections, $\sim 0.03 \mathrm{~mL}$ each) was applied to the skull along the incision line.

Rats were placed in a stereotaxic apparatus (David Kopf) and body temperature was maintained at $36^{\circ} \mathrm{C}-37^{\circ} \mathrm{C}$ by an electrical heating blanket and additional insulating material wrapped around the animal. The right eye was closed and the left eye was kept open using a small hemostat to allow presentation of visual stimuli. Tear-gel was applied to keep the open eye lubricated. The skull was exposed, and small skull holes were drilled to allow access to $\mathrm{V} 1$ (anterior-posterior $[\mathrm{AP}]+7.5 \mathrm{~mm}$, lateral [L] $-3.5 \mathrm{~mm}$, ventral [V] -0.5 to $-1.0 \mathrm{~mm})$, and the ipsilateral LGN ([AP] $+3.9 \mathrm{~mm}$ to $+4.3 \mathrm{~mm}$, [L] $-3.9 \mathrm{~mm}$, [V] $-4.6 \mathrm{~mm}$ to $-5.0 \mathrm{~mm})$. A recording electrode $(125-\mu \mathrm{m}$ diameter Tefloninsulated steel wire) was lowered onto the surface of V1, $\sim 0.5 \mathrm{~mm}$ below the surface of the skull. Additional skull holes (contralateral to the electrodes) over the prefrontal cortex and cerebellum allowed for the insertion of ground and reference connections, respectively. All stereotaxic measurements were based on the anatomical work of Paxinos and Watson (2007).

\section{Electrophysiology}

\section{Visual evoked potentials (VEPS)}

Following the final adjustments of electrodes, visual stimuli were presented to the animals. A liquid crystal display (LCD) computer monitor (model Acer, size $44 \mathrm{~cm}$ ) was positioned $55 \mathrm{~cm}$ in front and $8 \mathrm{~cm}$ away from the midline of the animal, toward the open eye. DirectRT Precision Timing Software (version 2004.3.0.27, Empirisoft) was used to present the visual stimuli and trigger the electrophysiological recording set-up. Visual stimuli (bitmap images, resolution $621 \times 480$ ) used for VEP experiments were identical (size, shape) to those used during behavioral training.

The VEPs elicited in V1 in response to visual stimuli were differentially recorded against a reference screw placed in the bone overlying the cerebellum. The signal was amplified (Grass P511 amplifiers, Grass Instruments Division, Astro-Med, Inc.; half amplitude filters set at $0.3 \mathrm{~Hz}$ and $1 \mathrm{kHz})$, digitized $(10 \mathrm{kHz}$, $\mathrm{AD}$ Instruments $16 / \mathrm{s}$ system), and stored for subsequent offline analysis using the PowerLab system (PowerLab/16 s system with ML 180 Stimulus Isolator, ADInstruments).

For all trained and control rats, VEPs were elicited by successively presenting one of three different visual stimuli $(\mathrm{P}+, \mathrm{P}-$, novel; see above; $5000 \mathrm{msec}$ duration each). A further, task-naïve group that had not received behavioral training was also presented with three visual stimuli, which obviously were all novel to these animals. In all cases, a total of 300 stimulus presentations occurred (100 presentations/stimulus; order randomized between rats, but the same sequence repeated for each animal). For all experiments, visual cues were also presented with a barrier placed between the computer monitor and the open eye of the animal. This control procedure was included to ascertain that VEPs were trigged by the image being projected onto the retina, rather than some other electrical or magnetic interference.

\section{Thalamocortical LTP and LTD experiments}

Immediately after completion of the VEP recordings, different rats were assigned to receive either TBS or LFS of the LGN in order to characterize LTP and LTD induction in V1. A concentric bipolar stimulation electrode (Rhodes Medical Instruments, Series 100, David Kopf) was placed in the LGN, while the recording electrode remained in V1, an electrode arrangement equivalent to that used by Heynen and Bear (2001) in their characterization of LTP between LGN and V1 under in vivo (anesthetized) conditions. The fPSPs recorded under these conditions largely reflect excitatory current sinks originating in layers II/III of V1 (Heynen and Bear 2001). Final placements of LGN and V1 electrodes were adjusted to elicit maximal fPSP amplitude and augmenting responses (at 100-msec interpulse intervals) in V1 in response to LGN stimulation. An input/output curve was generated by applying single LGN pulses ( $0.2 \mathrm{msec}$ duration) at intensities of 0.1 to $1.0 \mathrm{~mA}$, in $0.1 \mathrm{~mA}$ increments. The stimulation intensity eliciting $50 \%-60 \%$ of maximal fPSP amplitude was used for the remainder of the experiment. Baseline fPSPs were recorded every $30 \mathrm{sec}$ for $30 \mathrm{~min}$, or until a stable baseline had been established (95\%-105\% of average baseline fPSP amplitude). Baseline fPSP recordings were followed by either TBS or LFS of the LGN in separate animals. TBS consisted of stimulation bursts (five pulses per burst at $100 \mathrm{~Hz}$ ), with bursts repeated at $5 \mathrm{~Hz}$ for a total of 10 bursts (pulse duration and intensity same as above). These parameters were chosen based on previous work demonstrating robust, near-maximal LTP with this stimulation protocol (Dringenberg et al. 2007). LFS consisted of 900 bursts (three pulses per burst at $20 \mathrm{~Hz}$ ) repeated at $1 \mathrm{~Hz}$, a protocol known to induce intermediate-term depression of V1 synapses in vivo (Hager and Dringenberg 2010). Recordings of fPSPs (every $30 \mathrm{sec}$ ) continued for $1 \mathrm{~h}$ following either induction protocol, after which TBS or LFS was repeated and recordings continued for another $60 \mathrm{~min}$.

At the end of the experiment, rats were perfused through the heart with $10 \%$ formalin, their brains were removed, and standard histological techniques were used to verify all electrode placements. Data obtained with inaccurate placements were excluded from the data analyses.

\section{Data analysis}

Data are expressed as mean \pm S.E.M. In cases where repeatedmeasures tests were performed within the same subject (i.e., VEPs elicited by $\mathrm{P}+, \mathrm{P}-$, and a novel visual stimulus in the same animal), S.E.M. was calculated, but note that they reflect variance across subjects (larger or smaller VEP amplitude elicited by the 
same visual stimulus in different rats), while the statistical analysis was based strictly on a within-subjects comparison (i.e., VEP amplitude elicited by the three visual cues in the same rat). As such, S.E.M.s are not informative with regard to estimating the variance of dependent variables in data sets based on strictly within-subjects, repeated-measure designs (for detailed review, see Belia et al. 2005). All analyses were performed using SPSS (version 17.0, SPSS Inc.). Behavioral data were examined comparing the number of days to reach successful completion of the task among subgroups using one-way ANOVA. For multiple visual cue discrimination, a repeated-measures $t$-test was used to examine differences in training time for the two sets of visual cues.

For each animal, VEPs elicited by the three different visual stimuli (P+, P-, novel, 100 VEPs/stimulus) were averaged. The maximum amplitude of the positive peak of the VEP was computed offline using Scope Software (version 3.6.5, ADInstruments, Inc.). These amplitude values obtained for each visual stimulus were then compared within each group of animals (trained, control, naïve) using separate repeated-measures ANOVAs.

For LTP and LTD experiments, the maximal amplitude of the negative-going peak of each fPSP was computed offline. The amplitude values for each animal were then averaged over 10min intervals, and these averages were normalized by dividing them by the mean baseline amplitude of that animal. Mixed twoway ANOVAs were used to examine differences in fPSP amplitude between groups and over the time course of the experiment. Where statistically appropriate, ANOVAs were followed up by post-hoc, pairwise comparisons or simple effects.

\section{Acknowledgments}

The work described here was funded by the Natural Sciences and Engineering Research Council of Canada (NSERC) to H.C.D.

\section{References}

Bao S, Chan VT, Merzenich M. 2001. Cortical remodeling induced by activity of ventral tegmental dopamine neurons. Nature 412: 79-83.

Belia S, Fidler F, Williams J, Cumming G. 2005. Researchers misunderstand confidence intervals and standard error bars. Psychol Methods 10: 389-396.

Clapp WC, Eckert MJ, Teyler TJ, Abraham WC. 2006. Rapid visual stimulation induces $N$-methyl-D-aspartate receptor-dependent sensory long-term potentiation in the rat cortex. Neuroreport 17: 511-515.

De Villers-Sidani E, Chang EF, Bao S, Merzenich MM. 2007. Critical period window for spectral tuning defined in the primary auditory cortex (A1) in the rat. J Neurosci 27: 180-189.

Dringenberg HC, Hamze B, Wilson A, Speechly W, Kuo MC. 2007. Heterosynaptic facilitation of in vivo thalamocortical long-term potentiation in the adult rat visual cortex by acetylcholine. Cereb Cortex 17: 839-848.

Epp J, Keith JR, Spanswick SC, Stone JC, Prusky GT, Sutherland RJ. 2008. Retrograde amnesia for visual memories after hippocampal damage in rats. Learn Mem 15: 214-221.

Fournier GN, Semba K, Rasmusson DD. 2004. Modality- and region-specific acetylcholine release in the rat neocortex. Neurosci 126: 257-262.

Frenkel MY, Sawtell NB, Diogo ACM, Yoon B, Neve RL, Bear MR. 2006. Instructive effect of visual experience in mouse visual cortex. Neuron 51: $339-349$.

Gu Q. 2003. Contributions of acetylcholine to visual cortex plasticity. Neurobiol Learn Mem 80: 291-301.

Hager AM, Dringenberg HC. 2010. Assessment of different induction protocols to elicit long-term depression (LTD) in the rat visual cortex in vivo. Brain Res 1318: $33-41$.
Hanover JL, Huang ZJ, Tonegawa S, Stryker MP. 1999. Brain-derived neurotrophic factor overexpression induces precocious critical period in mouse visual cortex. J Neurosci 19: RC40 (1-5).

Heynen AJ, Bear MF. 2001. Long-term potentiation of thalamocortical transmission in the adult visual cortex in vivo. J Neurosci 21: 9801-9813.

Jiang B, Huang ZJ, Morales B, Kirkwood A. 2005. Maturation of GABAergic transmission and the timing of plasticity in visual cortex. Brain Res Rev 50: $126-133$

Kang JI, Vaucher E. 2009. Cholinergic pairing with visual activation results in long-term enhancement of visual evoked potentials. PLoS One 4: e5995. doi: 10.1371/journal.pone.0005995.

Karmarkar UR, Dan Y. 2006. Experience-dependent plasticity in adult visual cortex. Neuron 52: 577-585.

Kilgard MP, Merzenich MM. 1998. Cortical map reorganization enabled by nucleus basalis activity. Science 279: 1714-1718.

Kirkwood A, Rioult MG, Bear MF. 1996. Experience-dependent modification of synaptic plasticity in visual cortex. Nature 381: $526-528$

Kuo MC, Dringenberg HC. 2008. Histamine facilitates in vivo thalamocortical long-term potentiation in the mature visual cortex of anesthetized rats. Eur J Neurosci 27: 1731-1738.

Kuo MC, Dringenberg HC. 2009. Short-term ( 2 to 5 h) dark exposure lowers long-term potentiation (LTP) induction threshold in rat primary visual cortex. Brain Res 1276: 58-66.

Massey PV, Phythian D, Narduzzo K, Warburton EC, Brown MW, Bashir ZI. 2008. Learning-specific changes in long-term depression in adult perirhinal cortex. J Neurosci 28: 7548-7554.

Paxinos G, Watson C. 2007. The rat brain in stereotaxic coordinates, 6th edition. Academic Press, New York.

Prusky GT, Reidel C, Douglas RM. 2000a. Environmental enrichment from birth enhances visual acuity but not place learning in mice. Behav Brain Res 114: $11-15$.

Prusky GT, West PWR, Douglas RM. 2000b. Experience-dependent plasticity of visual acuity in rats. Eur J Neurosci 12: 3781-3786.

Rasmusson DD. 2000. The role of acetylcholine in cortical synaptic plasticity. Behav Brain Res 115: 205-218.

Rioult-Pedotti MS, Friedman D, Donoghue JP. 2000. Learning-induced LTP in neocortex. Science 290: 533-536.

Rioult-Pedotti MS, Donoghue JP, Dunaevsky A. 2007. Plasticity of the synaptic modification range. J Neurophysiol 98: 3688-3695.

Rodriguez R, Kallenbach U, Singer W, Munk MH. 2010. Stabilization of visual responses through cholinergic activation. Neurosci 165: 944-954.

Rogan MT, Staubli UV, LeDoux JE. 1997. Fear conditioning induces associative long-term potentiation in the amygdala. Nature 390: $552-553$.

Ross RM, McNair NA, Fairhall SL, Clapp WC, Hamm JP, Teyler TJ, Kirk IJ. 2008. Induction of orientation-specific LTP-like changes in human visual evoked potentials by rapid sensory stimulation. Brain Res Bull 76: 97-101.

Sale A, Maya Vetencourt JF, Medini P, Cenni MC, Baroncelli L, De Pasquale R, Maffei L. 2007. Environmental enrichment in adulthood promotes amblyopia recovery through a reduction of intracortical inhibition. Nat Neurosci 10: 679-681.

Teyler TJ, Jamm JP, Clapp WC, Johnson BW, Corballis MC, Kirk IJ. 2005. Long-term potentiation of human visual evoked responses. Eur J Neurosci 21: 2045-2050.

Weinberger NM. 2003. The nucleus basalis and memory codes: Auditory cortical plasticity and the induction of specific, associative behavioral memory. Neurobiol Learn Mem 80: 268-284.

Weinberger NM. 2004. Specific long-term memory traces in primary auditory cortex. Nature 5: 279-290.

Whitlock JR, Heynen AJ, Shuler MG, Bear MF. 2006. Learning induces long-term potentiation in the hippocampus. Science 313: 1093-1097.

Yashiro K, Philpot BD. 2008. Regulation of NMDA receptor subunit expression and its implications for LTD, LTP, and metaplasticity. Neuropharm 55: 1081-1094.

Received February 15, 2010; accepted in revised form June 10, 2010. 


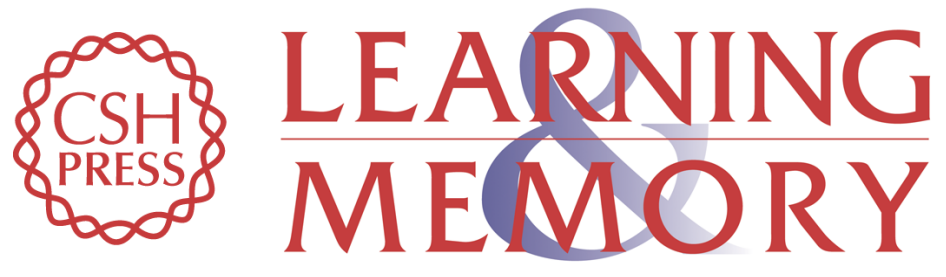

\section{Training-induced plasticity in the visual cortex of adult rats following visual discrimination learning}

Audrey M. Hager and Hans C. Dringenberg

Learn. Mem. 2010, 17:

Access the most recent version at doi:10.1101/Im.1787110

References This article cites 33 articles, 7 of which can be accessed free at:

http://learnmem.cshlp.org/content/17/8/394.full.html\#ref-list-1

License

Email Alerting Receive free email alerts when new articles cite this article - sign up in the box at the Service top right corner of the article or click here. 